\title{
Terapias de errores con aprendizaje móvil y gamificación: estudio comparativo en español de los negocios
}

\author{
Error Therapy with Mobile Learning and Gamification: \\ A Comparative Study in Business Spanish \\ Terapia dos erros com aprendizagem móvil e gamificação: \\ estudo comparativo em espanhol dos negócios \\ Carmen Mateo Gallego ${ }^{1}$ \\ Guadalupe Ruiz Yepes ${ }^{2}$
}

\section{Resumen}

Este artículo de investigación compara los resultados de un análisis sobre la evolución de la interlengua escrita de dos grupos de aprendientes de español como lengua extranjera (ELE). Los participantes realizaron cuatro redacciones a lo largo de un semestre seguidas respectivamente de una sesión de terapia de errores. El grupo de prueba realizó las terapias mediante la plataforma online Kahoot, que introduce la gamificación y permite la metodología mobile learning, mientras que el grupo de control realizó actividades tradicionales en papel. Las profesoras señalaron los errores de las redacciones mediante el criterio minimal marking (Haswell, 1983) y seguidamente los estudiantes procedieron a su autocorrección. Aunque con los dos métodos se obtuvieron resultados positivos, el grupo de prueba muestra una evolución más notable en cuanto a la reducción del número de errores de cada escrito.

\section{Palabras clave}

interlengua; aprendizaje móvil; gamificación; minimal marking; terapia de errores

\section{Abstract}

This research paper compares the results of an analysis of the evolution of written interlanguage in two groups of learners of Spanish as a foreign language (SFL). The participants wrote four essays throughout a semester, each one followed by an error-therapy session, respectively. The test group did the therapies through online platform Kahoot, which introduces gamification and allows the mobile learning methodology, while the control group did traditional activities on paper. The teachers marked the mistakes in the texts using the minimal marking criterion (Haswell, 1983), and the students then proceeded to correct their own errors. Although both methods had positive results, the test group shows a more remarkable evolution in terms of reducing the number of errors in each text.

\section{Keywords}

interlanguage; mobile learning; gamification; minimal marking; error therapy

\section{Resumo}

Este artigo de pesquisa compara as descobertas de uma análise sobre a evolução da interlíngua escrita de dois grupos de aprendentes de espanhol como língua estrangeira (ELE). Os participantes realizaram quatro textos ao longo do semestre seguidos cada um de uma sessão de terapia de erros. 0 grupo de prova realizou a terapia por meio da plataforma online Kahoot, que introduz a gamificação e possibilita a metodologia mobile learning, enquanto o grupo de controle realizou atividades tradicionais em papel. As professoras assinalaram os erros das escritas por meio do critério minimal marking (Haswell, 1983) e em seguida os estudantes auto-corrigiram seus textos. Ainda que as duas metodologias obtiveram resultado positivos, o grupo de prova apresentou uma evolução mais evidente ao respeito da redução no número de erros de cada texto.

\section{Palavras-chave}

interlíngua; aprendizagem móvil; gamificação; minimal marking; terapia de erros

Artículo recibido el 25 de julio de 2017 y aprobado el 10 de enero de 2018

1 Heilbronn University (Alemania). Correo electrónico: Carmen.mateo-gallego@hs-heilbronn.de

2 Heilbronn University (Alemania). Correo electrónico: Guadalupe.ruiz-yepes@hs-heilbronn.de 


\section{Introducción}

Aprender una lengua extranjera sin cometer errores de manera sistemática es prácticamente imposible, ya que estos están ligados intrínsecamente al proceso de aprendizaje (Dulay, Burt y Krashen, 1982, p. 138). Independientemente de si se trata de la lengua materna o una lengua extranjera, las producciones orales y escritas de los aprendientes presentarán incorrecciones propias de los distintos estadios del aprendizaje en el que se encuentran.

Desde el surgimiento de la teoría del análisis de errores de Pit Corder (1967) cambió la concepción negativa del error en el aula de segundas lenguas para convertirse en una fuente valiosa de información que revela las dificultades individuales de los aprendientes. Este cambio de paradigma dio lugar a numerosos estudios en el ámbito del español como lengua extranjera (ELE), que contribuyeron a un mejor conocimiento de la tipología y las causas de las incorrecciones de los aprendientes. Igualmente, surgieron propuestas didácticas para el tratamiento del error que contribuían a una correcta escritura a largo plazo.

Este trabajo se centra en un estudio comparativo con dos grupos de aprendientes universitarios alemanes de ELE. Los participantes de ambos grupos realizan cuatro redacciones a lo largo de un semestre, las cuales constituyen el corpus de este estudio. Los errores que cometen se tratan en clase de manera terapéutica siguiendo distintos métodos didácticos. El grupo de control realiza las terapias de errores mediante ejercicios tradicionales ${ }^{3}$ en papel, mientras que el grupo de prueba utiliza Kahoot, una plataforma online que introduce el elemento lúdico en el aprendizaje. Esta plataforma permite crear ejercicios de respuesta múltiple para ser llevados a cabo en clase mediante el smartphone, introduciendo así la metodología mobile learning.

Después de realizar las terapias de errores, los aprendientes llevan a cabo una autocorrección de su escrito siguiendo las pautas del método minimal

3 Con el adjetivo tradicional nos referimos a actividades que se realizan sin el apoyo de ningún aparato tecnológico ni internet. marking (Haswell, 1983). En este procedimiento, el docente señala en el margen del texto únicamente el número de errores encontrados en cada una de las líneas del escrito. Posteriormente, el alumno recibe su texto marcado y tiene 15 minutos para identificar y subsanar sus errores sin ayuda del docente.

Por último, los estudiantes rellenan un diario de aprendizaje que consta de una parte dedicada a la autorreflexión y otra a la autoevaluación. El grupo de control trabaja con un diario en papel mientras que el grupo de prueba lo hace mediante la plataforma ILIAS, de tipo learning management system (LMS), muy extendida en el ámbito universitario alemán.

Los resultados de la comparación y el seguimiento de ambos grupos nos permite sacar conclusiones sobre la evolución de los errores de ambos grupos, teniendo en cuenta la naturaleza de los métodos utilizados: el tradicional y plataformas didácticas online con gamificación y mobile learning. Además, es posible determinar los errores que se repiten con más frecuencia y la utilidad del método minimal marking para la autocorrección.

\section{Estado de la cuestión}

\section{La importancia del error en la adquisición de segundas lenguas}

En el contexto del behaviorismo de los años 1950, se desarrolló la teoría del análisis contrastivo. El fundamento de este enfoque era un aprendizaje impecable de las lenguas sin errores en su producción. Además, el behaviorismo culpaba a la lengua materna de todos los errores cometidos en las producciones de los aprendientes en la lengua extranjera.

En el marco histórico de esta visión tan negativa del error, Pit Corder publicó un trabajo titulado The significance of learner's errors (1967) que dio paso a una nueva concepción del error en el aprendizaje de segundas lenguas. Este nuevo enfoque no sitúa únicamente a la lengua materna en el foco de todos los errores de los aprendientes, sino que expone que las causas del error son de diversa naturaleza; entre ellas, la propia lengua meta, ya que el aprendiente, al no tener el dominio necesario del idioma, formula 
hipótesis sobre las estructuras, las reglas gramaticales, y el vocabulario que la rigen. También otorga importancia a las estrategias psicolingüísticas implicadas en el aprendizaje, al concepto de la gravedad del error y a la tipología de los mismos.

A partir del análisis de errores (AE), surge también el concepto de interlengua (IL) (Selinker, 1972). Este término hace alusión al sistema lingüístico de los aprendientes de una lengua extranjera que, entre otras particularidades, se caracteriza también por contener incorrecciones. Con base en el surgimiento del AE, diversos autores han postulado clasificaciones de los errores de los aprendientes, con énfasis en el estadio de la IL, los motivos de la incorrección y la capacidad de autocorregirse. Este último factor, la autocorrección, constituye junto con la autorreflexión y la autoevaluación los elementos principales de la metodología de este trabajo.

A la hora de subsanar errores, los factores de autocorrección y autonomía desempeñan un papel muy importante. Si los estudiantes reciben un texto corregido por el docente, no se fijarán en sus incorrecciones ya que considerarán que la tarea está concluida. Si no se trabajan los errores en clase y se les hace conscientes de ellos a través de la autocorrección, no le prestarán la atención suficiente como para poder aprender de ellos.

Edge (1989), James (1998) y Marín (2013) crearon clasificaciones de errores de aprendientes de segundas lenguas basándose en el grado de autocorregibilidad que presentan. Edge (1989) llama faltas a las incorrecciones que el aprendiente es capaz de autocorregirse. Los errores son los que, a pesar de marcar dónde se encuentra la incorrección, el aprendiente no tiene la capacidad suficiente de autocorregirse. La tercera tipología que propone consiste en el nivel del alumno y le da el nombre de tentativa. Para Edge (1989) este tipo de incorrección aparece cuando el aprendiente intenta expresar algo para lo que todavía carece de la competencia necesaria en la lengua extranjera.

Esta clasificación coincide con la de James (1998) en el concepto de falta, ya que ambos afirman que son incorrecciones que se pueden autocorregir.
Sin embargo, James (1998) apunta que las faltas se autocorrigen sin ayuda externa, es decir, sin que el docente indique la incorrección. Por tanto, introduce una tercera tipología a la que denomina equivocación, que se corresponde con las anomalías que el aprendiente se puede autocorregir, pero solo a través de una indicación explícita del docente. Por último, ambos autores coinciden en que el concepto de error es una incorrección para la cual el alumno todavía no goza del nivel necesario que requiere su identificación y autocorrección correspondiente.

Marín (2013) simplifica las clasificaciones anteriores y toma en consideración únicamente los conceptos de falta y error. Es decir, no hace alusión al nivel del alumno ni a la actuación del docente mediante la señalización explícita del lugar del error.

Dado que en este trabajo marcamos el número de errores de cada escrito mediante el método minimal marking (Haswell, 1983) para proceder a la autocorrección del aprendiente, nos basamos en la clasificación de Marín (2013), que defiende que las anomalías de la IL solo pueden ser autocorregibles (faltas) y no autocorregibles (errores). Haswell (1983) afirma, además, que con su método, los estudiantes de inglés como lengua extranjera son capaces de autocorregirse más de la mitad de las incorrecciones $(61,1 \%)$, independientemente de la tipología a la que pertenecen.

En este trabajo se utilizará el término error para referirnos a todo tipo de anomalías de la IL en general indistintamente de la nomenclatura empleada en las clasificaciones existentes. Además, al igual que Haswell (1983), hemos seguido el criterio de excluir del estudio las incorrecciones relacionadas con los signos de puntuación. Según él, este tipo de errores no constituyen una parte esencial en el desarrollo de la destreza escrita de los estudiantes de una lengua extranjera (p. 600). De esta manera, adaptándonos a su criterio, podemos comparar el porcentaje de errores subsanados en este estudio basado en aprendientes de ELE con el porcentaje que logaron corregir los aprendientes de inglés como lengua extranjera que participaron en su estudio. 
En el ámbito de ELE, se han realizado numerosos trabajos sobre errores a partir de la década de 1990, que toman como referencia la lengua materna de los aprendientes. Por ejemplo, los trabajos de Vázquez (1991) y De Alba (2009) se centran en los errores de la IL de aprendientes alemanes y han contribuido especialmente a la categorización lingüística de las incorrecciones cometidas y a un mejor conocimiento de los errores léxico-semánticos.

\section{Cómo tratar el error}

En cuanto al tratamiento del error que se debe hacer en clase, muchos autores coinciden en que el docente debe crear una atmósfera positiva y distendida en la que se elogie el trabajo bien hecho. Muñoz-Basols (2004) emplea técnicas basadas en el humor a la hora de corregir errores y utiliza frases como "An error a day keeps the teacher away" o "A la abuela Dolores le gustan los errores" (p. 32). Blanco Picado (2002) se muestra igualmente a favor de emplear el refuerzo positivo y afirma que la sanción constante por parte del docente puede llevar al alumno a desarrollar sus estrategias de defensa y a no querer expresarse en la lengua meta por miedo al error. Esta conducta solo lleva a la frustración y al desinterés por el idioma (p.15).

Para subsanar los errores de aprendientes de segundas lenguas, han sido muchas las actividades sugeridas por diversos autores a lo largo de las últimas décadas. Los métodos han seguido dos criterios: el preventivo y el terapéutico. Con preventivo, se intenta que los estudiantes eviten cometer errores antes o durante el proceso de escritura. Por ejemplo, Cassany (1993) propone unas listas de control para reparar la escritura de los estudiantes de ELE en las que se recuerdan los puntos más importantes a tener en cuenta.

Por otro lado, el método terapéutico se centra en el tratamiento del error una vez que ha ocurrido. En este trabajo, nos hemos decantado por ese método y lo llevamos a cabo mediante actividades de distinta índole y metodología didáctica.

A la hora de tematizar terapias de errores, los estudios pasan de un plano exclusivamente lingüístico al área de la didáctica (De Alba, 2009). Hasta la fecha, se han propuesto actividades terapéuticas que contribuyen a una corrección del error a largo plazo como trucos, consejos y métodos mnemónicos (Vázquez, 1991). También se ha constatado en estudios recientes que las dinámicas sociales y el aprendizaje con tareas colaborativas en el aula de idiomas son aptos para este efecto. Tardo y Socorro (2014) llevaron a cabo un experimento con alumnos canadienses en el que demuestran la eficacia de las técnicas de animación sociocultural y de actividades en grupos para reducir errores.

\section{Mobile learning y gamificación}

El uso del dispositivo móvil para el aprendizaje de idiomas recibe el nombre de MALL (mobile assisted language learning) y hasta el año 2009, gran parte de las investigaciones sobre esta metodología se fundamentaban en proyectos y experimentos cuya principal función era el envío de sMs (Jordano y Pareja, 2016, p. 26). El rápido avance de las tecnologías de la información ha hecho posible un gran desarrollo en este ámbito. Hoy en día, gracias a la aparición de los smartphones, el aprendizaje se produce de manera ubicua, espontánea y flexible debido a su fácil portabilidad.

Al contrario que el aprendizaje de lenguas asistido por ordenador, conocido como computer assisted lenguage learning (CALL), el surgimiento del MALL se produjo en contextos informales y se fue integrando poco a poco en los contextos de enseñanza formal (Jordano y Pareja, 2016, p. 30). En este trabajo, hacemos uso de MALL en las clases presenciales para las terapias de errores del grupo de prueba, combinado además con gamificación. Este término fue acuñado por Deterding, Khaled, Nacke y Dixon (2011) que afirman que la gamificación es: "el uso de las mecánicas de juego en entornos ajenos al juego" (p. 1). Según Zichermann y Cunningham (2011), el uso de las dinámicas de juego con fines didácticos crea lo que se denomina en psicología estado de flujo, que consiste en el incremento de varios factores como la atención, el rendimiento y el esfuerzo. 
Ahora bien, como señalan Foncubierta y Rodríguez (2014), la gamificación en sí no asegura que se cumplan estos objetivos, ya que, para que una tarea gamificada tenga éxito, el docente tiene que abordar el ejercicio de tal manera que el estudiante aprenda y que aporte un reto cognitivo (p. 3). Si se lleva a cabo una actividad en la que aparecen insignias, puntos y ganadores, pero carece de una progresión didáctica que plantee un reto en el aprendiente, la actividad no contribuirá a fomentar el estado de flujo, sino que se traducirá en desinterés.

En el aula de ELE, como en el aula de otros idiomas y de otras materias, la metodología de la gamificación ya se usaba antes de que se acuñara este término. "Los profesores de español ya sabíamos usar el juego como instrumento de aprendizaje, y así hemos hecho nuestros 'juegos de la oca' y 'nuestros trivial adaptados al mundo ELE"' (Foncubierta y Rodríguez, 2014, p. 2). El elemento novedoso de la gamificación es la posibilidad de llevarla a cabo mediante herramientas y plataformas digitales creadas específicamente para ese fin.

\section{Estudios similares}

Existen estudios que coinciden con el presente trabajo o bien en la comparación de métodos tradicionales de enseñanza frente al aprendizaje electrónico o e-learning, o bien en la comparación de los errores de distintos grupos de aprendientes de ELE. Sin embargo, no hay constancia de estudios en los que se haya comparado la evolución de la IL de dos grupos de aprendientes de ELE tratando los errores mediante el método tradicional de enseñanza, y plataformas online con gamificación y mobile learning.

Martínez et al. (2012) llevaron a cabo un estudio similar con dos grupos de estudiantes universitarios para comprobar la eficacia del método learning by doing, o aprender haciendo, expresión para el aprendizaje centrado en la experiencia y la interacción, frente a la metodología de enseñanza tradicional en el ámbito universitario. En la metodología learning by doing se incluyeron igualmente plataformas online y herramientas digitales. Las notas finales de los estudiantes que siguieron esta metodología fueron más altas que con los que se empleó el método tradicional.

Entre los estudios sobre la evolución de la IL de aprendientes de ELE se encuentra el de Liu (2012), que comparó los errores de estudiantes chinos de ELE procedentes de la Universidad de La Habana (Cuba). El primer grupo lo constituían graduados universitarios mientras que el segundo estaba compuesto por estudiantes de primer año de carrera de la misma titulación. El estudio dio a conocer que el grupo de los no titulados cometía más errores relacionados con la concordancia de género y número, mientras que los titulados cometían errores de concordancia, solo de número, y errores de omisión del artículo.

Bueno (2014) comparó la evolución de la IL de aprendientes estadounidenses de ELE. Para este estudio, el factor principal era la lengua materna y los conocimientos de otras lenguas. Por tanto, la autora separó a los aprendientes en cuatro grupos. El objetivo del estudio era observar la evolución del uso de los pronombres personales átonos dentro de los cuatro grupos. Los resultados muestran que el grupo que más errores presenta es el conformado por hablantes nativos de inglés sin conocimiento de otras lenguas.

\section{Metodología}

Este estudio es de corte experimental, comparativo y basado en datos cualitativos y cuantitativos aportados por los errores encontrados en las muestras escritas. La fase experimental se llevó a cabo durante un semestre con dos grupos de estudiantes alemanes de ELE de nivel intermedio de las titulaciones de Economía y Turismo. La carga lectiva del español en el plan de estudios es de cuatro horas pedagógicas a la semana.

El corpus está compuesto por las redacciones de los estudiantes, escritas a mano en clase, sin ayuda de fuentes de consulta. La temática del escrito fue la misma para los dos grupos y se reveló de manera espontánea en el momento de proceder a la escritura. El tiempo de realización de la redacción fue de 15 minutos y la extensión de la muestra escrita 
está entre 150 y 160 palabras. Este procedimiento se llevó a cabo en clase con un intervalo de 4 semanas.

En la primera muestra escrita, los participantes escribieron sobre las razones por las que estudian español, en la segunda, tuvieron que dar su opinión sobre las ventajas y desventajas del Brexit. La tercera trató de un comentario de texto sobre la reducción de la jornada laboral por paternidad, y la última correspondió con el ejercicio de expresión escrita del examen final. En esta última muestra, los estudiantes escribieron sobre el ramsonware, o secuestros de móviles mediante la descarga de aplicaciones. $\mathrm{Al}$ igual que en el texto anterior, se trató de un comentario sobre una noticia actual.

Para realizar un seguimiento de los participantes en la investigación, se entregó un perfil el primer día de clase con preguntas sobre las variables que, según Ellis (2005), influyen en el proceso de aprendizaje de una lengua extranjera: edad, lengua materna y conocimiento de otras lenguas extranjeras. Ambos grupos están formados por estudiantes con edades comprendidas entre 19 y 25 años, cuya lengua materna es el alemán. El grupo de control tiene un nivel B1.2, y el grupo de prueba tiene un nivel de español B1.1. Todos los componentes de ambos grupos afirman poseer un dominio de inglés de nivel $\mathrm{C} 1$.

Además de figurar esta información sobre las variables que influyen en el proceso de aprendizaje, se anotó en el perfil de cada estudiante la cantidad de errores que cometieron en cada redacción y la categoría a la que pertenecen, según la clasificación de Fernández López (1997). Esta autora se basa en el criterio lingüístico y distingue entre cuatro subtipos de errores (pp. 44-48). Por un lado, los errores léxicos que afectan tanto a la forma como al significado, como el uso de un significante próximo al que se quiere expresar en una situación comunicativa determinada como: "*tengo que aprender para el examen", en vez de "tengo que estudiar para el examen"; el uso incorrecto de préstamos de distintas lenguas, como: “* promenades” o "^advantajes” o un registro no apropiado a la situación comunicativa.

Los errores gramaticales están relacionados mayoritariamente con los verbos; por ejemplo, el contraste de los pasados y el uso del subjuntivo como en las frases siguientes: "^ cuando viví en México, iba en bicicleta" o "^quiero que vienes a mi casa". Además de los errores de índole verbal, dentro de esta clase se engloban también los que afectan al uso de las categorías, las preposiciones, la estructura oracional y la subordinación.

El siguiente grupo lo constituyen los errores discursivos que afectan a la coherencia del texto y a al uso incorrecto de los conectores, y por último, los errores gráficos se corresponden con las tildes, el uso de mayúsculas y minúsculas, la confusión de fonemas, como es el caso de "ellos dos son *nuvios" y de grafemas, como la palabra "*extranjero".

Después de la fase de autocorrección de los estudiantes, mediante el método minimal marking, se anotó la cantidad de errores que subsanaron en el periodo de 15 minutos. Esta vez estaba permitido el uso de fuentes de consulta. Pasado este tiempo, se recolectaron de nuevo los textos escritos y se procedió a una segunda ronda de corrección por parte del docente, que según el fundador del método (Haswell, 1983) consiste en lo siguiente: "as papers are returned to me I review the corrections, mending those errors left undiscovered, miscorrected or newly generated" (p. 601). En la sesión de clase posterior a la terapia y la autocorrección, se les entregó la versión final de su escrito. Una vez culminado este paso, se procede a la fase de autorreflexión y autoevaluación mediante un diario de aprendizaje.

\section{Preguntas de investigación}

Este trabajo se centra en las siguientes preguntas:

a. ¿Qué errores se dan en la IL escrita de aprendientes de ELE de nivel intermedio?

b. ¿Cómo influye la metodología empleada en el tratamiento del error?

c. ¿Qué errores persisten, aunque se traten de manera terapéutica?

d. ¿Supone la aplicación de MALL una mejora en la evolución de la IL respecto al método tradicional? 


\section{Fase de terapias de errores}

La plataforma Kahoot introduce elementos típicos de entornos de juego, como música de fondo, acumulación de puntos por cada respuesta correcta y puntos extra por responder con rapidez. Después de cada pregunta, aparece en la pantalla el número de aciertos y de equivocaciones, así como un listado de los alumnos con mejor puntuación.

La figura 1 muestra una visión general del funcionamiento de esta plataforma. A la derecha, aparece un dispositivo móvil que se corresponde con la vista del alumno. En pantalla aparecen dos símbolos para cada una de las posibles respuestas. A la izquierda, aparece la pantalla principal que se proyecta en el aula con el enunciado del ejercicio.

Mediante esta plataforma se trabajaron los errores más problemáticos de cada muestra escrita del grupo de prueba. El grupo de control realizó actividades en papel sin ayuda soportes electrónicos.

La última fase de este ciclo de corrección de errores culminó con la confección de un diario de aprendizaje. Esta fase se fundamentó en el propósito de hacer a los estudiantes dedicar tiempo a reflexionar sobre sus errores y a autoevaluar su propio trabajo. Además, fomenta la capacidad de aprender a aprender. Según Trujillo (2013), un diario de aprendizaje "es, por excelencia, el mecanismo de autoevaluación cualitativa más interesante” (p. 5).

Dado que un diario de aprendizaje es una herramienta que debe servir únicamente como fuente de información acerca del proceso de aprendizaje del alumno, realizamos las preguntas en alemán. De esta manera, queda asegurado que el idioma no supone ningún impedimento a la hora de expresarse.

Los alumnos de los dos grupos contestaron a las mismas preguntas, lo único que cambió en este caso fue también el formato de la encuesta. El grupo de control lo hizo en papel, mientras que el de prueba empleó la plataforma ILIAs.

Las preguntas fueron las siguientes:

a. ¿Qué he aprendido de la terapia de errores?

b. ¿Qué es lo que me resulta más difícil al escribir un texto?

c. ¿Qué ejercicios me ayudan a no cometer errores al escribir una redacción?

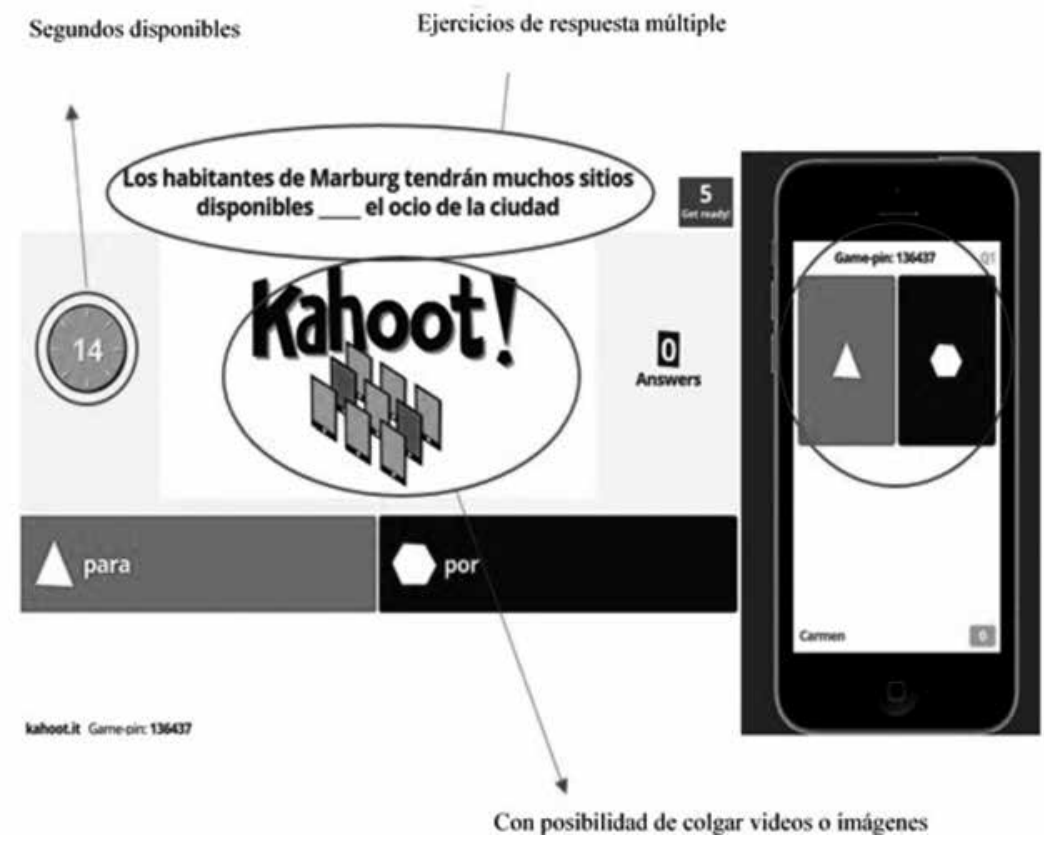

Figura 1. Ejemplo de una actividad con Kahoot

Fuente: www.Kahoot.it 
d. ¿Qué temario debería repasar de nuevo?

e. ¿Qué puedo hacer para mejorar la expresión escrita?

f. ¿Cuántos puntos le daría a mi redacción en una escala del 1 al 10 ?

\section{Resultados}

\section{Primera prueba escrita}

El primer escrito se llevó a cabo el primer día de clase en ambos grupos; los participantes debían responder a la pregunta de por qué estudian español. En el grupo de control se recolectaron 15 redacciones y se encontraron 135 errores en total. Por tanto, la media de los errores del grupo de control en el primer escrito fue de 9 por estudiante.

En el grupo de prueba, los errores fueron considerablemente más numerosos. Se encontraron en total 320 en las 20 redacciones que se entregaron en clase. Por tanto, la media en este caso fue de 16 errores por estudiante.

A continuación, expondremos los cinco errores que más se repitieron en el primer escrito y la tipología a la que pertenecen:

Grupo de control

- Tildes inexistentes o mal colocadas.

- Confusión entre el imperfecto y el indefinido.

- Personas del verbo mal conjugadas; confusión entre la primera y la tercera persona del singular del pretérito indefinido.

- Confusión entre las preposiciones por y para.

- Concordancias de género: mayoritariamente artículo y sustantivo o sustantivo y adjetivo.

Grupo de prueba

- Tildes inexistentes o mal colocadas.

- Confusión entre el imperfecto y el indefinido.

- Concordancias de género: mayoritariamente artículo y sustantivo o sustantivo y adjetivo.

- Personas del verbo mal conjugadas; confusión entre la primera y la tercera persona del singular del pretérito indefinido.

- Confusión entre las preposiciones pory para.
Los dos errores más frecuentes coinciden en ambos grupos. Estos son: el uso incorrecto de las tildes, y la confusión entre el imperfecto y el indefinido. Según la clasificación de Fernández López (1997), corresponden a la categoría de errores gráficos y gramaticales respectivamente. En el grupo de control, los errores que más se repiten después de estos dos son de índole gramatical: la confusión de personas del verbo y el uso incorrecto de preposiciones. Por último, el quinto error es de origen léxico, ya que se trata de las concordancias de género entre el artículo y el sustantivo o el sustantivo y el adjetivo. Este mismo error se repite en el grupo de prueba y constituye, además, el tercer grupo más abundante.

La confusión entre la primera y la tercera persona del singular en la conjugación del pretérito indefinido ocupa en el grupo de prueba la cuarta posición, mientras que en el grupo de control ocupó la tercera. Por último, el error gramatical que afecta a la confusión de preposiciones, especialmente al uso de por y para, ocupa en el grupo de prueba el quinto puesto mientras que en el de control está en la cuarta posición.

Como la tipología de los errores que cometieron los estudiantes de ambos grupos fue muy similar, las sesiones destinadas al tratamiento del error se centraron en la misma temática. En los dos grupos se repasaron todos los errores cometidos y se ahondó en las reglas del acento ortográfico y el contraste de los pasados con especial atención entre el pretérito imperfecto y el indefinido. El grupo de prueba trabajó los errores con la plataforma Kahoot utilizando el dispositivo móvil y el grupo de prueba realizó ejercicios en papel.

A la hora de proceder a la autocorrección, los estudiantes del grupo de control se autocorrigieron el $88,8 \%$ de los errores en total con una media de 8 errores por estudiante. En el grupo de prueba, se autocorrigieron una media de 7 errores por estudiante, es decir, un 43,7 \% del número total de errores.

Finalmente, en la siguiente sesión de clase, entregamos las redacciones con todos los errores corregidos y resolvimos dudas posibles sobre la corrección final. Después, se concedieron 5 minutos para contestar a las preguntas del diario de aprendizaje. 
Las respuestas sobre lo aprendido en las terapias de errores fueron muy diversas, pero todos se basaron en algún aspecto de sus errores al que hasta ahora no le habían prestado la atención suficiente. Por ejemplo, algunos se percataron de que los errores que más cometen se relacionan con la elección de los tiempos verbales, o con que no todas las palabras que tienen tilde en singular la siguen teniendo en plural.

En cuanto a lo que les resulta más difícil a la hora de escribir textos, en el grupo de control y en el de prueba las respuestas fueron también diversas, aunque comparten algunos aspectos comunes. Muchos de los aprendientes de ambos grupos coinciden en la falta de vocabulario al escribir, el desconocimiento de la correcta posición del acento ortográfico, los tiempos del pasado y el subjuntivo. La fase de autorreflexión contiene opiniones muy diversas sobre los ejercicios que más les ayudan a evitar los errores. Por un lado, hay opiniones contrarias a los ejercicios de rellenar huecos, algunos piensan que son una buena opción para evitar errores, mientras otros piensan que son poco prácticos a la hora de escribir un texto libremente.

Los puntos que le darían a su texto los participantes del grupo de control en una escala del $1 \mathrm{al}$ 10 es de 7,4 de media, mientras que en el grupo de prueba este valor baja a una media de 5,65.

\section{Segunda prueba escrita}

El segundo escrito se realizó dejando un tiempo de cinco semanas con respecto del primero. El tema escogido en este caso fue "las ventajas y desventajas del Brexit", y tanto los tiempos como los procedimientos a seguir en la corrección y en las terapias fueron los mismos.

En el grupo de control se recolectaron 19 muestras escritas de los aprendientes con un total de 234 errores, lo que da lugar a una media de 12,3 errores por estudiante. En el grupo de prueba fueron 17 las muestras escritas recolectadas con un total de 258 errores, con una media de 15,1 .

A continuación, expondremos los cinco errores que más se repitieron en ambos grupos:

\section{Grupo de control}

a. Tildes: mal colocadas o inexistentes.

b. Confusión entre las preposiciones pory para.

c. Subjuntivos inexistentes.

d. Concordancia de género y de número entre artículos y sustantivos y sustantivos y adjetivos.

e. Confusión de fonemas.

La tipología de los errores que se dan en el segundo escrito del grupo de control es muy similar a la de los del primero, después de haberlos tratado en clase anteriormente. El error más frecuente sigue siendo el uso de las tildes, de índole gráfica. Los errores gramaticales ocupan el segundo y el tercer puesto, ya que son de naturaleza preposicional o verbal, como el uso incorrecto del subjuntivo. Los que ocupan el cuarto y quinto puesto son de índole léxica. Se vuelve a repetir el error de concordancia de género y número, y aparece uno que anteriormente no se presentaba: la confusión de fonemas, de tipo gráfico. Los casos más comunes que ilustran este error son: "^los inglesos", "^La Unión Europía” y "^la monida de Inglaterra".

Grupo de prueba

a. Subjuntivos inexistentes.

b. Concordancia de género y número entre artículos y sustantivos, y sustantivos y adjetivos.

c. Confusión entre las preposiciones pory para.

d. Tildes inexistentes.

e. Confusión entre el uso del imperfecto y el indefinido.

En el grupo de prueba, el error más encontrado es el uso incorrecto del subjuntivo, de carácter gramatical; seguido de la concordancia de género y número, de carácter léxico. El tercero, esta vez, consiste en la confusión entre por y para, de índole gramatical; el cuarto puesto lo ocupa el uso incorrecto de las tildes, que se corresponde con la categoría gráfica, y por último, de carácter gramatical, vuelve a aparecer la confusión de tiempos del pasado. 
Los errores del segundo escrito de ambos grupos se consideran fosilizables ${ }^{4}$ y guardan paralelismos con los errores del primer escrito. La única diferencia esta vez es que, en el grupo de control, el quinto puesto lo ocupan errores léxicos; mientras que, en el grupo de prueba, el quinto error más frecuente es de índole gramatical. La falta de tildes vuelve a aparecer en los dos grupos, a pesar de haber sido tratados en la terapia de errores del primer escrito.

Esta vez, la media del número de errores de los estudiantes del grupo de control aumentó a 12,3 frente a los 9 de la primera muestra escrita. En el grupo de prueba, disminuyó ligeramente, de 16 en el primer escrito a 15,1 en el segundo. En general, la tipología de los errores del segundo escrito fue más heterogénea que en el primero; por tanto, fue más complicado establecer los errores más frecuentes.

En los dos grupos se trabajó la diferencia entre las preposiciones por y para y el uso del subjuntivo. Además, el tercer error que trató el grupo de prueba fue la concordancia de género y número entre artículos y sustantivos, y sustantivos y adjetivos, mientras que en el grupo de control se trataron nuevamente las tildes. Una vez más, en el grupo de control se usaron ejercicios sobre papel y en el grupo de prueba se utilizó la gamificación con Kahoot para el tratamiento de los errores.

Culminada la fase destinada al tratamiento del error, se procedió a la autocorrección de 15 minutos. Durante este tiempo, el grupo de control subsanó el $54,4 \%$ de las incorrecciones, con una media de 6,7 por estudiante, mientras que en el grupo de prueba fueron $44,3 \%$ del total. La media de errores corregidos por los estudiantes es la misma que en el grupo de control, es decir 6,7.

Una vez recibieron las versiones finales de sus redacciones, se pasó a la realización del diario de aprendizaje. Ambos grupos afirman que lo que más dificultades ocasionó al escribir sobre el Brexit fue la carencia de vocabulario en el ámbito político e

4 Nos basaremos en la definición de errores fosilizables de Fernández López (1995): "son aquellos que se repiten en fases sucesivas y que ofrecen una mayor resistencia, ya sea por la complejidad misma de la estructura, por un problema de interferencia o por cualquier otra clase de contaminación" (p. 212). institucional. Como factor extralingüístico, se nombra además la falta concentración y el factor tiempo. En cuanto a los ejercicios que les ayudan a mejorar la escritura, la mayoría coincide también en leer más en español y en realizar más ejercicios de escritura libre. En la autoevaluación, el grupo de control le da a su escrito una nota media de 7,4, mientras que en grupo de prueba, este valor se reduce a 5,4.

\section{Tercera prueba escrita}

Esta vez se procedió a un comentario de texto sobre un tema de actualidad. Para ello, se seleccionó una noticia de un periódico que exponía el caso de un hombre que fue despedido de su puesto de trabajo al solicitar una reducción de la jornada laboral para cuidar de sus hijos. La noticia se adaptó según el nivel y se redujo a seis líneas para que contuviera únicamente la información más relevante de la noticia. Después de la lectura, debían contestar a la siguiente pregunta: “¿Piensas que los hombres deben tener el mismo derecho que las mujeres a una reducción de la jornada laboral para cuidar de sus hijos?".

En el grupo de control se recolectaron 14 redacciones con 125 errores en total, lo que equivale a una media de 8,9 errores por estudiante. Esta vez, la media se redujo frente los 12,3 de la segunda muestra escrita.

En el grupo de prueba, se recolectaron 13 redacciones, y se cometieron 137 errores en total, es decir, una media de 10,5 por estudiante. En comparación con el segundo escrito, los errores del grupo de prueba también se han reducido notablemente.

La tipología de los errores de los dos grupos conserva bastante similitud con los cometidos en los escritos anteriores:

Grupo de control

a. Subjuntivos inexistentes y en menor frecuencia también se encuentran subjuntivos superfluos.

b. Tildes diacríticas en los siguientes casos: $\mathrm{mi} /$ mí, si/sí, donde/dónde, quien/quién, como/ cómo. En menos casos también se encontraron tildes superfluas como en "^aunque". 
c. Concordancias de género y de número entre artículos y sustantivos, y sustantivos y adjetivos.

d. Confusión de fonemas: “`defenir”, "^resultir”.

e. Confusión entre las preposiciones por y para.

Grupo de prueba

a. Subjuntivos inexistentes.

b. Concordancias de género y de número entre artículos y sustantivos, y sustantivos y adjetivos.

c. Confusión entre las preposiciones por y para

d. Confusión de fonemas para el mismo grafema: "*quando", "* quatro".

e. Tildes inexistentes.

El error gramatical referente al uso del subjuntivo vuelve a ser el más frecuente en ambos grupos, con la particularidad de que en el grupo de control también se dan subjuntivos superfluos. El segundo error gramatical que se sigue repitiendo a lo largo de los tres escritos es la confusión entre las preposiciones por y para.

Los errores gráficos también siguen estando representados dentro del grupo de los más frecuentes de ambos grupos a través de las tildes. Esta vez, en el grupo de control también han aparecido tildes superfluas. El siguiente error gráfico presente en la IL escrita de los estudiantes es la confusión de fonemas y de grafemas.

En la fase de tratamiento del error, el grupo de control trabajó las tildes y el uso del subjuntivo, mientras que, en el grupo de prueba, se trató nuevamente la problemática del subjuntivo, la concordancia de género y número y el uso de las preposiciones por y para usando ejercicios tradicionales y Kahoot respectivamente.

En la fase de autocorrección, el grupo de control fue capaz de subsanar el 52,8 \% de los errores cometidos, con una media de 4,7 por estudiante. Este porcentaje aumentó en el grupo de prueba a un $62,8 \%$ y media de 6,6 errores subsanados, lo que indica una mejora frente al 44,3\% del escrito anterior.
En el diario de aprendizaje, la nota media que se otorga el grupo de control es de 7 . Los estudiantes señalan también que les pareció difícil la identificación de sus propios errores, como evitar algunos que aparecen por la falta de concentración. La nota de las redacciones del grupo de prueba aumenta en este caso ligeramente a 5,7 puntos sobre 10 .

\section{Resultados finales}

La última redacción que constituye el corpus de este estudio coincidió con la parte de expresión escrita del examen final de la asignatura. Al igual que el escrito anterior, se basó en un comentario de texto sobre una noticia de actualidad. En este caso, la noticia versaba sobre los secuestros de móviles mediante la descarga de aplicaciones y de nuevo tuvieron que escribir entre 150 y 160 palabras. Los dos grupos escribieron basándose en la información que contenía el texto y expresando su opinión sobre ese tema.

Se tuvieron en cuenta únicamente las redacciones de los estudiantes que habían asistido regularmente a clase y habían participado en las sesiones de autocorrección y de terapias de errores. Por tanto, se tuvieron en cuenta 18 exámenes en el grupo de prueba y 16 en el de control. El número de errores cometidos en el grupo de prueba fue de 185 , lo que equivale a una media de 10,27 errores por estudiante. En el grupo de control se cometieron 179 , lo que equivale a una media de 11,18 errores.

En cuanto a la tipología de los errores cometidos, los más abundantes tanto en el grupo de control como en el de prueba han sido esta vez los de naturaleza gráfica, como la confusión de grafemas para el mismo fonema, por ejemplo: "^quando", o confusión de fonemas: "* ordinador" $y$ “* desimpleo". Además de estos, que esta vez han ocupado el primer puesto, los demás errores que se han repetido son los mismos que surgieron a lo largo del estudio. En el grupo de prueba se han dado los siguientes errores por orden de frecuencia:

a. Falta de concordancia de género y de número.

b. Uso incorrecto del subjuntivo. 
c. Confusión de las preposiciones por y para.

d. Ausencia de tildes.

En el grupo de control los errores más frecuentes son:

a. Uso incorrecto del subjuntivo.

b. Ausencia de tildes.

c. Concordancia de género y número.

d. Confusión de las preposiciones por y para.

Las figuras 2 y 3 dan una idea de la evolución del número de errores de ambos grupos a lo largo del semestre, así como de los autocorregidos justo después de las terapias.

Tanto el número de errores cometidos como el de autocorregidos han seguido una evolución distinta en los dos grupos. En el grupo de control, el desarrollo de los errores ha sido heterogéneo. En el primer escrito y en el tercero se cometieron 3 errores menos de la media por estudiante, en comparación con la segunda redacción y el examen final.

Como se observa en la figura 2 , en el grupo de control se han ido reduciendo paulatinamente los errores en cada uno de los escritos. Si comparamos el primer y el último escrito, se puede observar que la media de errores por estudiante ha disminuido de 16 a 6 errores.

En cuanto a la autocorrección, se puede observar que en ambos grupos el número de errores autocorregidos disminuye en cada uno de los escritos. En el grupo de control, los estudiantes se autocorrigieron una media de 8 errores en la primera redacción,

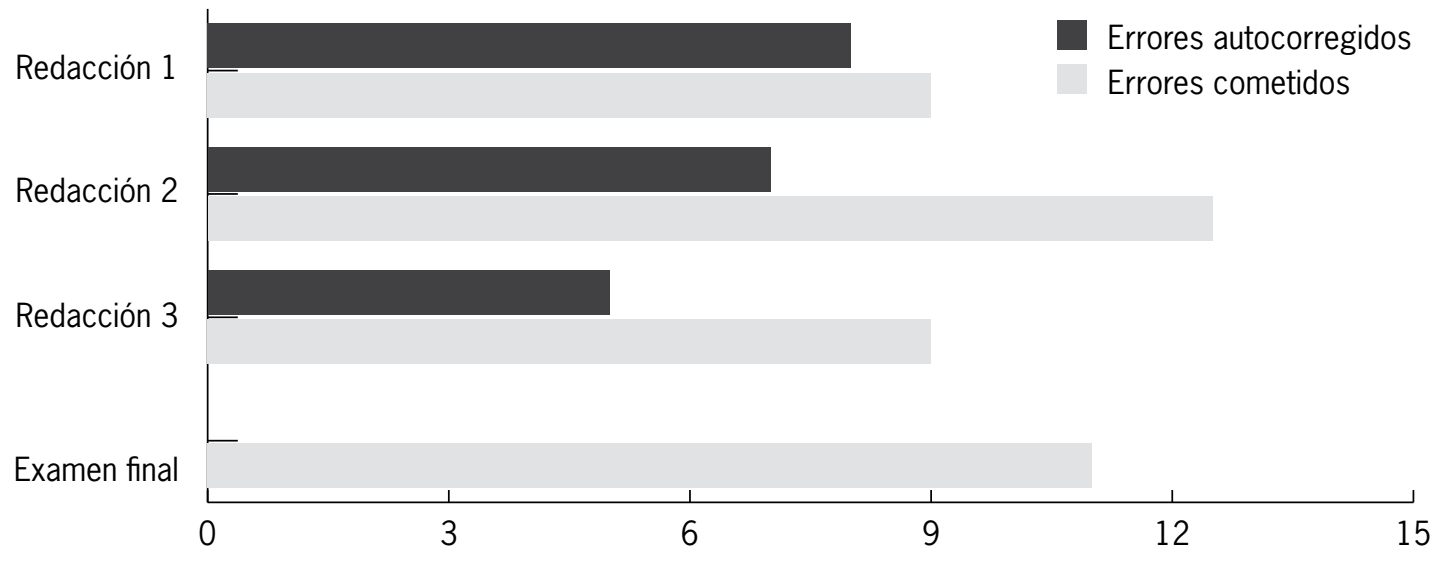

Figura 2. Evolución de los errores del grupo de control

Fuente: elaboración propia.

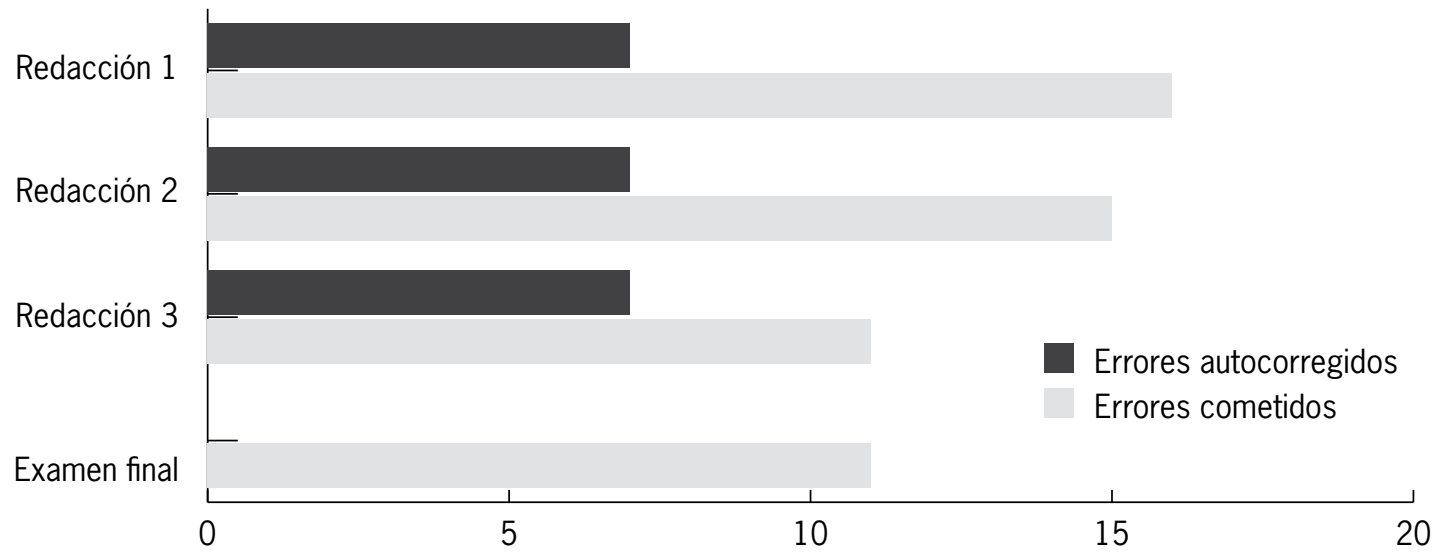

Figura 3. Evolución de los errores del grupo de prueba

Fuente: elaboración propia. 
un valor que se va reduciendo en los demás escritos hasta llegar a una media de 4,75 en la tercera. En el grupo de prueba, este descenso es muy leve, ya que en el primer escrito se autocorrigieron 7 errores de media, en el segundo 6,75 y en el tercero 6,60.

\section{Conclusiones}

La tipología de los errores de ambos grupos ha sido muy similar a lo largo del estudio. Además, el hecho de que vuelvan a aparecer en escritos posteriores después de haberlos tratado mediante terapias, incluso en más de una ocasión, muestra que son altamente fosilizables.

Los errores que se han analizado en este estudio coinciden con los de análisis sobre errores de estudiantes de ELE de distintas lenguas maternas y niveles. Por ejemplo, Kočman (2011) analizó los errores de 480 redacciones de aprendientes croatas de niveles A1, A2 y B1. Los principales errores que encontró esta autora fueron: el uso de las tildes, confusión en el pretérito indefinido y el pretérito imperfecto, ser y estar, uso del subjuntivo y confusión entre la primera y la tercera persona del singular del indefinido. En cuanto a este último, algunos autores coinciden en que se trata de una incorrección generalizada como De la Cava y Sesmilo (2006) y otros que defienden que se trata de errores aislados como Jelić y Guć (2015). En este estudio, la confusión de la primera y la tercera persona se ha dado también, aunque de manera minoritaria y únicamente en el primer escrito. Por tanto, aquí lo consideraremos igualmente un error aislado.

La utilización del verbo ser en lugar del verbo estar también se ha repetido a lo largo de este trabajo. Aunque no ha llegado a ser uno de los errores más frecuentes de las redacciones de ambos grupos, lo consideramos fosilizable debido a su persistencia.

En conclusión, los errores fosilizables de los aprendientes de ELE se deben a las reglas que no existen en otras lenguas y aparecen independientemente del nivel. Como ejemplo de estos errores, se puede citar la existencia de tildes, la coexistencia de los verbos ser y estar en oposición a otras lenguas en las que solo existe un único verbo para los dos casos, la existencia de subjuntivo o el contraste entre el indefinido y el imperfecto, así como el uso de las preposiciones por y para en oposición a la lengua alemana, que solo tiene la preposición für. Todos estos errores son típicos de los aprendientes de español porque son rasgos característicos de esta lengua.

La tercera conclusión está relacionada con las fases de autocorrección y con el diario de aprendizaje. Durante el proceso en el que se ha desarrollado este estudio, y tomando en cuenta los escritos de los participantes de los dos grupos, el porcentaje de errores autocorregidos a través del método minimal marking ha sido de un 57,8 \%. Una cifra muy parecida a la de Haswell (1983), quien afirma que los estudiantes de inglés como lengua extranjera son capaces de autocorregirse el $61,1 \%$ de los errores totales cometidos.

El diario de aprendizaje ha tenido mejor aceptación en el grupo de control que en el grupo de prueba. En general, las respuestas dadas por el grupo de prueba han sido mucho más escuetas, algunas se han dejado sin responder o simplemente se han respondido mediante un "no lo sé". Algunos estudiantes escribieron comentarios positivos sobre el uso de Kahoot para llevar a cabo las terapias de errores.

Los participantes del grupo de control han dado respuestas más precisas sobre su proceso de aprendizaje y sus aptitudes a la hora de escribir en español. Las respuestas que se han dado en el diario de aprendizaje han seguido un proceso más profundo de reflexión en comparación con el grupo de prueba.

La última conclusión está relacionada con los métodos utilizados en las terapias. Aunque los resultados han sido positivos en ambos grupos, muestran una evolución distinta. Mientras que en el grupo de prueba se ha observado una reducción paulatina del número de errores en cada escrito, en el grupo de control no ha seguido ningún patrón cronológico. La reducción de errores ha dependido más bien de la temática del escrito. El número de errores es más bajo en el primero y en el tercer escrito, mientras que ha aumentado en el segundo y en el examen final.

Por tanto, este estudio muestra cómo las terapias de errores llevadas a cabo mediante la plataforma 
Kahoot han contribuido a unos resultados más positivos y homogéneos frente al método tradicional. El tratamiento del error con el dispositivo móvil a través de elementos típicos de la gamificación como música, recolección de puntos y alumnos ganadores resalta la idea de muchos autores como MuñozBasols (2004) y Blanco Picado (2002), que defienden que el componente lúdico y la actitud positiva frente al error contribuyen a una corrección a largo plazo.

\section{Referencias}

Blanco, A.I. (2002). El error en el proceso de aprendizaje. Cuadernos Cervantes de la Lengua Española, 38, 12-22.

Bueno, E. (2014). Estudio contrastivo de la interlengua de angloparlantes monolingües, bilingües y de perfil heritage estudiantes de ELE: a propósito de los pronombres átonos. (Trabajo de fin de Máster). Sevilla: Depósito de Investigación Universidad de Sevilla. Recuperado de http://hdl.handle.net/11441/23356

Cassany, D. (1993). Reparar la escritura. Didáctica de la corrección de lo escrito. Barcelona: Graó.

Corder, P. (1967). The Significance of Learners' Errors. International Review of Applied Linguistics in Language Teaching (IRAL), 5, 161-170.

De Alba, V. (2009). El análisis de errores ortográficos en aprendientes alemanes de español como lengua extranjera. Revista Philologia Hispalensis, 23,17-35. Recuperado de http://institucional.us.es/revistas/ philologia/23/02\%20Alba.pdf

De la Cava, P. y Sesmilo, A. (2006). Errores específicos en estudiantes eslavos (polacos y croatas) en las destrezas orales. En XVII Congreso Internacional de la Asociación del Español como lengua extranjera (ASELE). Universidad de Logroño, España.

Deterding, S., Khaled, R., Nacke, L. y Dixon, D. (2011). From game design element to gamefulness: defining gamification. En 15th International Academic MindTrek Conference: Envisioning Future Media. Tampere, Finlandia.

Dulay, H., Burt, M. y Krashen S. (1982). Language Two. Nueva York: Oxford University Press.

Edge, J. (1989). Mistakes and Correction. Londres: Longman.

Ellis, R. (2005). Instructed second language acquisition. A literature review. Wellington: Research Division of the Ministry of Education of New Zealand. Recuperado de http://www.mecd.gob.es/dctm/redele/ MaterialRedEle/Biblioteca/2006_BV_05/2006_ BV_05_04Ellis.pdf?documentId=0901e72b80e3a029

Fernández, S. (1995). Errores e interlengua en el aprendizaje del español como lengua extranjera. Didáctica, 7, 203-216.

Fernández, S. (1997). Interlengua y análisis de errores en el aprendizaje del español como lengua extranjera. Madrid: Edelsa.

Foncubierta, J.M. y Rodríguez, C. (2014). Didáctica de la gamificación en la clase de español. Madrid: Edinumen. Recuperado de https://www.edinumen. es/spanish_challenge/gamificacion_didactica.pdf

Haswell, R. (1983). Minimal Marking. College English Magazine, 45(6), 600-604. Recuperado de http:// users.ipfw.edu/wellerw/minimal_marking.pdf

James, C. (1998). Errors in Language Learning and Use. Exploring Error Analysis. Londres: Longman.

Jelić, A.B. y Guć, A. (2015). Análisis de errores verbales y su implicación comunicativa en la producción escrita de los alumnos croatas de ELE. Studia Romanica et Anglica Zagrabiensia (sRAZ LIX), 95-121.

Jordano, M. y Pareja, A. (2016). El aprendizaje de lenguas extranjeras mediante tecnología móvil en el contexto de la educación a distancia y combinada. Revista Iberoamericana de Educación a Distancia (RIED), 19, 25-40. Recuperado de http://www.dialogoseducativos.cl/revistas/n28/tardo.pdf

Kočman, A. (2011). Errores gramaticales comunes en alumnos croatas de español como segunda lengua en la producción escrita. Marco ELE, Revista de Didáctica de Español como Lengua Extranjera, 13, 1-12.

Lui, N. (2012). Análisis de errores y evolución de la interlengua de los estudiantes sinohablantes: esbozo de un estudio longitudinal. Barcelona: Depósito Digital de Documentos de la Universidad Autónoma de Barcelona. Recuperado de http://ddd.uab.cat/ record/106258

Martínez, A., Nuviala A., Pérez R., Grao, A., González, J.J., Porcel, A.M. y Tamayo, J. (2012). Estudio comparativo entre una metodología de aprendizaje tradicional respecto a una metodología de aprendizaje basada en el learning by doing para la consecución de competencias específicas. UPO INNOVA, Revista de Innovación Docente de la Universidad Pablo de Olavide (Sevilla), 1(1), 158-169.

Marín Serrano, F. (2013). Análisis y diagnóstico de errores en estudiantes de inglés como lengua 
extranjera. EXEDRA Revista Científica ESEC, 8, 182198. Recuperado de http://www.exedrajournal.com/ wp-content/uploads/2014/09/13.pdf

Muñoz-Basols, J. (2004). An error a day keeps the teacher away: Developing error Awareness an Error correction as learning strategies in the Spanish classroom. The Bulletin, 31-36.

Muñoz-Basols, J. (2005). Aprendiendo de los errores con la abuela Dolores. En I Congreso internacional de la Federación Internacional de Asociaciones de Profesores de Español (FIAPE). Toledo, España. Recuperado de https://www.mecd.gob.es/dam/ jcr:0305fc1a-24b5-490c-b27b-cacb1b1c2720/2005esp-05-35munoz-basols-pdf.pdf

Selinker, L. (1972): Interlanguage. International Review of Applied Linguistics in Language Teaching, 10, 16134141. Recuperado de https://www.degruyter.com/ downloadpdf/j/iral.1972.10.issue-1-4/iral.1972.10.14.209/iral.1972.10.1-4.209.xml

Tardo, Y. y Socorro, M.V. (2014). Experiencia didáctica del tratamiento del error en el aula de español como lengua extranjera a través de la animación sociocultural. Diálogos Educativos, 28, 101-118.

Trujillo, F. (2013). III.2 Diario de aprendizaje, rúbricas y portafolios. Madrid: Informe del Instituto Nacional de Tecnologías Educativas y Formación del Profesorado del Ministerio español de Educación, Cultura y Deporte. Recuperado de http://formacion. educalab.es/pluginfile.php/37234/mod_resource/ content/1/PDF/6_AbP_bloq3_u2.pdf

Vázquez, G. (1991). Análisis de errores y aprendizaje de español como lengua extranjera. Fráncfort del Meno: Peter Lang.

Zichermann, G. y Cunningham, C. (2011). Gamification by design. Sebastopol: O’Reilly Media.

\section{Para citar este artículo}

Mateo Gallego, C. y Ruiz Yepes, G. (2018). Terapias de errores con aprendizaje móvil y gamificación: estudio comparativo en español de los negocios. Folios, 48, 121-135. 